\title{
MODAL ANALYSIS OF BACK-TO-BACK PLANETARY GEAR: EXPERIMENTS AND CORRELATION AGAINST LUMPED-PARAMETER MODEL
}

\author{
Ahmed Hammami \\ Laboratory of Mechanics, Modeling and Production - National School of Engineers Sfax, Sfax, Tunisia and \\ Department of Structural and Mechanical Engineering, University of Cantabria, Santander, Spain \\ e-mail: ahmed_hammami@voila.fr
}

Alfonso Fernandez Del Rincon, Fernando Viadero Rueda

Department of Structural and Mechanical Engineering, University of Cantabria, Santander, Spain

e-mail: alfonso.fernandez@unican.es; fernando.viadero@unican.es

FAKher ChaAri, Mohamed Haddar

Laboratory of Mechanics, Modeling and Production - National School of Engineers Sfax, Sfax, Tunisia

e-mail:fakher.chaari@gmail.com; mohamed.haddar@enis.rnu.tn

\begin{abstract}
In order to characterize the dynamic behaviour of a back-to-back planetary gear, experimental and numerical modal analysis techniques are achieved. Rotational and translational modal deflections are highlighted. Natural frequencies are compared to the results from the lumped-parameter model. The modes are presented in the numerical studies in low-frequency and high-frequency bands. Distributions of modal kinetic and strain energies are studied.
\end{abstract}

Keywords: back-to-back planetary gears, modal analysis, lumped-parameter, impact test

\section{Introduction}

Gears are widely used in many power transmission applications and are characterized by their high efficiency. However, they can be subjected to severe operating conditions giving rise to dynamic problems. Bartelmus (2001) showed the influence of error mode parameters and the influence of clutch damping on the dynamic factor. Time varying stiffness and backlash, which have influence on the dynamic behaviour of the gearbox, are the main excitations of the model of Łuczko (2008) who studied the chaotic vibrations in one stage spur gear.

Planetary gears are compact kind of gears. They are excellent mechanisms for transmitting significant power with large speed reductions or multiplications. These kinds of gears are used in many fields of application like wind turbines, new generation aircraft engines, hybrid car transmissions. Back-to-back planetary gear configuration is compact with mechanical power circulation for economic and energy efficiency criteria.

Many researchers studied modal proprieties of planetary gears highlighting and the relationship between natural frequencies and parameters of the system. Cunliffe et al. (1974) focused on an analytical model of a planetary gear with a fixed carrier and characterised vibration modes. Botman (1976) studied the effects of planet pin loads on the eigen-frequencies using an eighteen degree of freedom system. August and Kasuba (1986) used a torsional model with nine degrees of freedom to determine torsional vibrations and dynamic loads in a basic planetary gear system. Kahraman (1993) and Sondkar and Kahraman (2013) analyzed respectively three dimensional modal deflection of a helical planetary gear and a double-helical planetary gear. Kahraman 
(1994) provided expressions for natural frequencies by using a rotational lumped-parameter model. Lin and Parker categorized rotational, translational, and planet modes and explained the unique modal proprieties of planetary gear with equal planet spacing (Lin and Parker, 1999) and unequal planet spacing (Lin and Parker, 2000). Tanna and Lim (2004) compared the modal frequencies of ring gears and idealized smooth rings. They quantified the frequency deviations in applying the simpler smooth ring solutions to represent the primary modal behaviour of ring gear structures. Wojnarowski et al. (2006) used the versatile graph theory to represent the structure of a planetary gear system and the gear modeling in order to achieve an eigen-vibration analysis of the gear system.

Wu and Parker (2008) considered the elastic deformation of the ring on the modal proprieties of a planetary gear with equal planet spacing and unequal planet spacing (Parker and $\mathrm{Wu}, 2010$ ). Eritenel and Parker (2009) presented the modal properties of three-dimensional helical planetary gears. Bu et al. (2012) developed a generalized dynamic model for a herringbone planetary gear train in order to investigate its modal properties. Vibration modes of compound planetary gears were studied by Kiracofe and Parker (2007) and Guo and Parker (2010). Cooley and Parker (2012, 2013) modeled the gyroscopic effects and studied the vibration properties of high-speed planetary gears.

There were few experimental studies to correlate the numerical modal analysis with experimental modal analysis. Singh et al., (2008) presented results of a comprehensive experimental and theoretical study under static condition to determine the influence of manufacturing tolerance on gear stresses and planetary load sharing. Kahraman and Vijayakar (2001) investigated the effect of internal gear flexibility under the quasi-static condition behaviour of a planetary gear set. Toda and Botman (1979) showed experimentally that planetary gear vibration resulting from the planet position error can be minimized by a proper indexing planet.

Velex et al. (1994) compared experimental results of a double helical epicyclic gear in its natural environment to a mathematical model. But some natural frequencies do not agreed between their model and experiments. Ericson and Parker (2013) applied experimental modal analysis techniques to characterize the planar dynamic behaviour of spur planetary gears and correlated these results against lumped parameter and finite element models.

This paper will discuss the modal properties of a special configuration of a two stage planetary gears mounted back-to-back. To achieve this, both numerical and experimental investigations are conducted. Experimental results are correlated against results from the lumped-parameter model. Modal characteristics are obtained and classified.

\section{Experimental setup}

\subsection{Description of the test bench}

A test bench composed of two identical planetary gear sets with the same gear ratio (Fig. 1) is developed at the University of Cantabria in Spain. It is composed of two identical planetary gear sets with the same gear ratio (Fig. 1).

The test bench is composed of a test gear set and a reaction gear set connected back-to-back: the sun gears of both planetary gear sets are connected through a common shaft, and the carriers of both planetary gear sets are connected to each other through a rigid hollow shaft (Fig. 1).

To load the system, masses are added to an arm fixed to the ring of the reaction gear set (Fig. 2).

\subsection{Excitation method}

In order to achieve the experimental modal analysis, an impact hammer is used. 


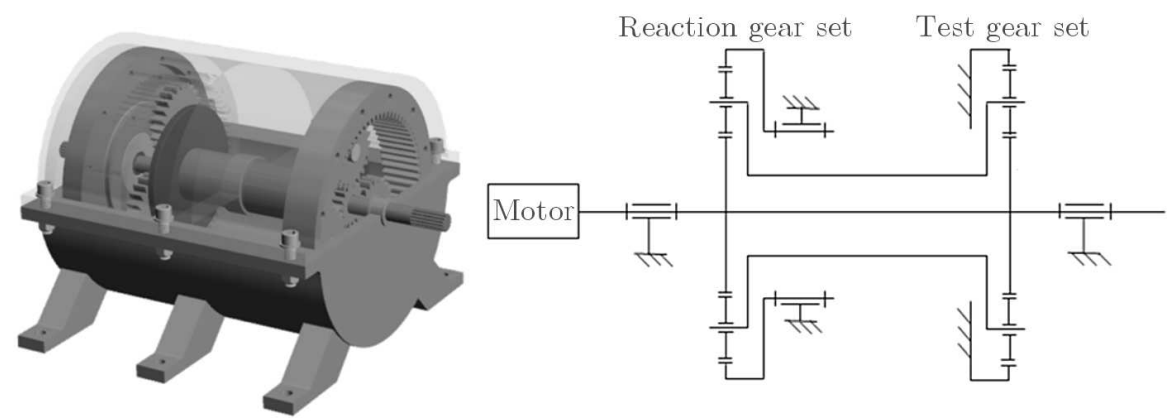

Fig. 1. Back-to-back layout as assembled in the bench

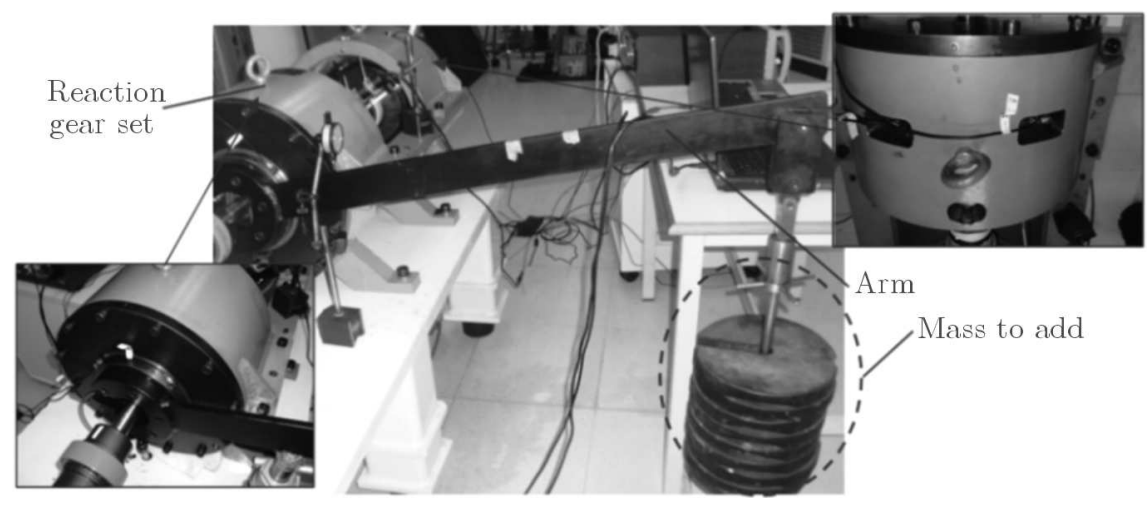

Fig. 2. Application of the external torque

The hammer model is PCB $086 \mathrm{~B} 03$ with a sensitivity of $2.15 \mathrm{mV} / \mathrm{N}$. A metal tip is used to study the response up to $2000 \mathrm{~Hz}$.

\subsection{Data acquisition}

Four tri-axial accelerometers mounted on the free ring and the fixed ring as well as the carrier measure the rotational and translational vibration of each body (Fig. 2).

The signals measured by the accelerometers will be acquired by an LMS SCADAS Mobile SCM01 system and the data will be processed with the software "LMS Test.Lab Impact test" to obtain the Frequency Response Function.

\section{Numerical model}

The model of the back-to-back planetary gear is based on the model developed by Lin and Parker (1999). The components are the ring $(r)$, sun $(s)$, planets $(1,2,3)$ and carrier $(c)$ which carries the planets as shown in Fig. 3.

The test ring and the test sun are respectively linked to the three planets of the test gear set via gear mesh stiffness $K_{r t 1}, K_{r t 2}, K_{r t 3}$ and $K_{s t 1}, K_{s t 2}, K_{s t 3}$. The same is on the reaction gear set, the reaction ring and the reaction sun are respectively linked to the three planets via gear mesh stiffness $K_{r r 1}, K_{r r 2}, K_{r r 3}$ and $K_{s r 1}, K_{s r 2}, K_{s r 3}$. The reaction ring which has the bearing stiffness $K_{r r x}$ and $K_{r r y}$ in the $x$ and $y$ directions is free and its torsional stiffness is $K_{r r u}$ whereas the test ring which has the bearing stiffness $K_{r t x}$ and $K_{r t y}$ is not exactly fixed but its torsional stiffness $K_{r t u}$ is very high. The planets of the reaction gear set and the test gear set have the bearing stiffness $K_{r p i x}$ and $K_{\text {tpiy }}$ in the $x$ and $y$ directions $(i=1,2$ or 3$)$. The test carrier and the reaction carrier have also the bearing stiffness respectively $K_{c t x}, K_{c t y}$ and $K_{c r x}, K_{c r y}$. The sun gears of both planetary gear sets are connected through a common shaft which has a torsional 


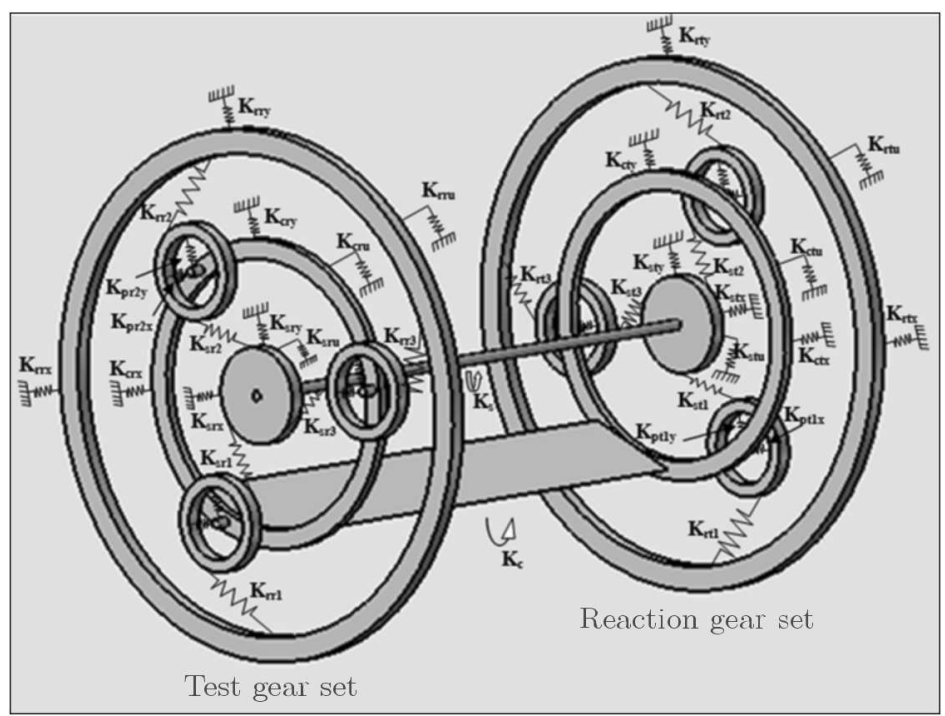

Fig. 3. Model of the planet gear

stiffness $K_{s}$. The carriers of both planetary gear sets are connected to each other through a hollow shaft which has a torsional stiffness $K_{c}$.

The equation of motion of the system for the back-to-back planetary gear with 3 planets is

$$
\mathbf{M} \ddot{\mathbf{q}}+\Omega_{c} \mathbf{G} \dot{\mathbf{q}}+\left[\mathbf{K}_{b}+\mathbf{K}_{M}-\Omega_{c}^{2} \mathbf{K}_{\Omega}\right] \mathbf{q}=\mathbf{F}(t)
$$

where $M$ is the mass matrix, $\mathbf{K}_{b}$ is the bearing stiffness matrix, $G$ and $\mathbf{K}_{\Omega}$ result from the high-speed carrier, $\mathbf{K}_{M}$ is the stiffness matrix and $\mathbf{F}(t)$ is the external force vector applied to the system. All these matrices are defined in appendix.

The planets in the test gear set and the reaction gear set are assumed identical and equally spaced. If the gyroscopic terms $\mathbf{G}, \mathbf{K}_{\Omega}$ and $\mathbf{F}(t)$ are neglected, the equation of motion will simplify to

$$
\mathbf{M} \ddot{\mathbf{q}}+\left[\mathbf{K}_{b}+\mathbf{K}_{M}\right] \mathbf{q}=\mathbf{0}
$$

$\mathbf{q}$ is the degree of freedom vector defined as the following

$$
\mathbf{q}=\left\{\begin{array}{l}
q_{r} \\
q_{t}
\end{array}\right\}
$$

$\mathbf{q}_{r}$ is the degree of freedom vector in the reaction gear set $(r)$ and $\mathbf{q}_{t}$ is the degree of freedom vector in the test gear set defined by

$$
\begin{aligned}
& \mathbf{q}_{r}=\left\{x_{c r}, y_{c r}, u_{c r}, x_{r r}, y_{r r}, u_{r r}, x_{s r}, y_{s r}, u_{s r}, \zeta_{1 r}, \eta_{1 r}, u_{1}, \zeta_{2 r}, \eta_{2 r}, u_{2 r}, \zeta_{3 r}, \eta_{3 r}, u_{3 r}\right\}^{\mathrm{T}} \\
& \mathbf{q}_{t}=\left\{x_{c t}, y_{c t}, u_{c t}, x_{r t}, y_{r t}, u_{r t}, x_{s t}, y_{s t}, u_{s t}, \zeta_{1 t}, \eta_{1 t}, u_{1 t}, \zeta_{2 t}, \eta_{2 t}, u_{2 t}, \zeta_{3 t}, \eta_{3 t}, u_{3 t}\right\}^{\mathrm{T}}
\end{aligned}
$$

The carrier, the ring and the sun translations $x_{i j}$ and $y_{i j}(i=c, r, s$ and $j=r, t)$ and planet translations $\zeta_{n j}$ and $\eta_{n j}(n=1,2,3$ and $j=r, t)$ are measured with respect to the rotational frame of reference $\mathbf{R}=\{\vec{i}, \vec{j}, \vec{k}\}$.

The rotational coordinates are $u_{r j}=r_{r j} \theta_{r j}$ for the reaction gear set $u_{t j}=r_{t j} \theta_{t j}$ and for the test gear set where $j=c, r, s, 1,2,3 . \theta_{r j}$ and $\theta_{t j}$ are the component rotation; $r_{r j}$ and $r_{t j}$ are the base radius for the sun, ring and planets and the radius of the circle passing through the planets centre for the carrier. 


\section{Comparative results}

In this part, results from the modal analysis on the back-to-back planetary gears are presented. Experimental and numerical modes are checked and compared. The modes are classified and studied in terms of modal kinetic energies and modal strain energies for each natural frequency. Finally, the effects of loading change (by adding mass) on natural frequencies and vibration modes are studied.

\subsection{Natural frequencies and vibration modes}

The natural frequencies and vibration modes are determined from equation of motion (3.2) and the associated eigen-value problem given by

$$
\omega_{i}^{2} \mathbf{M} \phi_{i}=\left[\mathbf{K}_{b}+\mathbf{K}_{M}\right] \phi_{i}
$$

where $\omega_{i}$ are the natural frequencies and $\phi_{i}$ are the vibration modes.

The rotational, translational and planet mode types were defined by Lin and Parker (1999):

- The rotational and translational modes are the vibration with natural frequencies having respectively the multiplicity $m=1$ and $m=2$

- The planet modes exist only if the number of planet $N>3$ and have multiplicity $m=N-1$.

In the test gear set and the reaction gear set, there are only three planets $(N=3)$. So, only rotational and translational modes will be expected when solving the eigen-value problem.

According to the energy distribution and component deflection, Cunliffe et al. (1974) and Ericson and Parker (2013) classified the natural frequencies into two categories according to the frequency band. The modes of the first band are called "bearing modes" or "fixture modes". The modes of the second band are called "tooth modes" or "gear modes".

The distinction between the fixture and gear modes is independent of the rotational and translational modes.

Table 1 shows the parameters of the model.

The natural frequencies are identified numerically and experimentally.

The impacts have been made on the arm of the free ring.

\subsubsection{Teeth modes}

For every test, we achieved ten impacts, and the acquisition system "LMS Test.Lab Impact test" averaged the results. Figure 4 represents the Frequency Response Function (FRF) in the fix ring.

Table 2 compares the natural frequencies determined from the impact test experiments and numerical model and shows the multiplicity of each mode from the analytical model.

Experiments show fourteen natural frequencies whereas the numerical model shows twelve natural frequencies composed of eight rotational modes and four translational modes. The natural frequencies $41 \mathrm{~Hz}, 182 \mathrm{~Hz}$ and $770 \mathrm{~Hz}$ appear only in the impulse test whereas the natural frequency $247 \mathrm{~Hz}$ appears only in the numerical model. The remaining natural frequencies agree within $11 \%$ as the maximum rate. There is only one identified natural frequency where the error reaches $30 \%$.

Figure 5 shows the modal deflection for the natural frequencies $192 \mathrm{~Hz}$ and $244 \mathrm{~Hz}$.

It is well noticed that all planets have the same motion and move in phase in the rotational modes $(118 \mathrm{~Hz}, 136 \mathrm{~Hz}, 244 \mathrm{~Hz}, 376 \mathrm{~Hz}, 387 \mathrm{~Hz}, 547 \mathrm{~Hz}$ and $550 \mathrm{~Hz})$. Besides, the carriers, the suns and the rings on the test and the reaction gear set have pure translation in the translation modes $(192 \mathrm{~Hz}, 288 \mathrm{~Hz}, 344 \mathrm{~Hz}$ and $536 \mathrm{~Hz})$. 
Table 1. Lumped-parameter values of the reaction and test planetary gear set and the shaft stiffness

\begin{tabular}{|c|c|c|c|c|}
\hline & Sun & Planet & Ring & Carrier \\
\hline \multicolumn{5}{|c|}{ Reaction planetary gear set } \\
\hline Teeth number & 16 & 24 & 65 & - \\
\hline Mass $[\mathrm{Kg}]$ & 0.485 & 1.225 & 28.1 & 3.643 \\
\hline Base diameter $[\mathrm{mm}]$ & 61.38 & 92.08 & 249.38 & 57.55 \\
\hline Moment of inertia $\left[\mathrm{Kg} \mathrm{m}^{2}\right]$ & $356 \cdot 10^{-6}$ & $2045 \cdot 10^{-6}$ & $697767 \cdot 10^{-6}$ & $21502 \cdot 10^{-6}$ \\
\hline Bearing stiffness $[\mathrm{N} / \mathrm{m}]$ & $8.8 \cdot 10^{7}$ & $3.5 \cdot 10^{6}$ & $2.1 \cdot 10^{7}$ & $4.8 \cdot 10^{8}$ \\
\hline Torsional stiffness $[\mathrm{N} / \mathrm{m}]$ & - & - & 0 & - \\
\hline Mesh stiffness $[\mathrm{N} / \mathrm{m}]$ & \multicolumn{2}{|c|}{$4.46 \cdot 10^{8}$} & \multicolumn{2}{|c|}{$6.28 \cdot 10^{8}$} \\
\hline \multicolumn{5}{|c|}{ Test planetary gear set } \\
\hline Teeth number & 16 & 24 & 65 & - \\
\hline Mass $[\mathrm{Kg}]$ & 0.485 & 1.225 & 28.1 & 3.643 \\
\hline Base diameter $[\mathrm{mm}]$ & 61.38 & 92.08 & 249.38 & 57.55 \\
\hline Moment of inertia $\left[\mathrm{Kg} \mathrm{m}^{2}\right]$ & $356 \cdot 10^{-6}$ & $2045 \cdot 10^{-6}$ & $697767 \cdot 10^{-6}$ & $21502 \cdot 10^{-6}$ \\
\hline Bearing stiffness $[\mathrm{N} / \mathrm{m}]$ & $8.8 \cdot 10^{7}$ & $3.5 \cdot 10^{6}$ & $2.1 \cdot 10^{7}$ & $4.8 \cdot 10^{8}$ \\
\hline Torsional stiffness $[\mathrm{N} / \mathrm{m}]$ & - & - & $7.9 \cdot 10^{6}$ & - \\
\hline Mesh stiffness $[\mathrm{N} / \mathrm{m}]$ & \multicolumn{2}{|c|}{$4.46 \cdot 10^{8}$} & \multicolumn{2}{|c|}{$6.28 \cdot 10^{8}$} \\
\hline \multicolumn{5}{|c|}{ Shaft stiffness } \\
\hline Torsional $[\mathrm{Nm} / \mathrm{rad}]$ & $3.73 \cdot 10^{4}$ & - & - & $8.38 \cdot 10^{5}$ \\
\hline Flexural & $4.9 \cdot 10^{5}$ & - & - & $1.1 \cdot 10^{7}$ \\
\hline
\end{tabular}

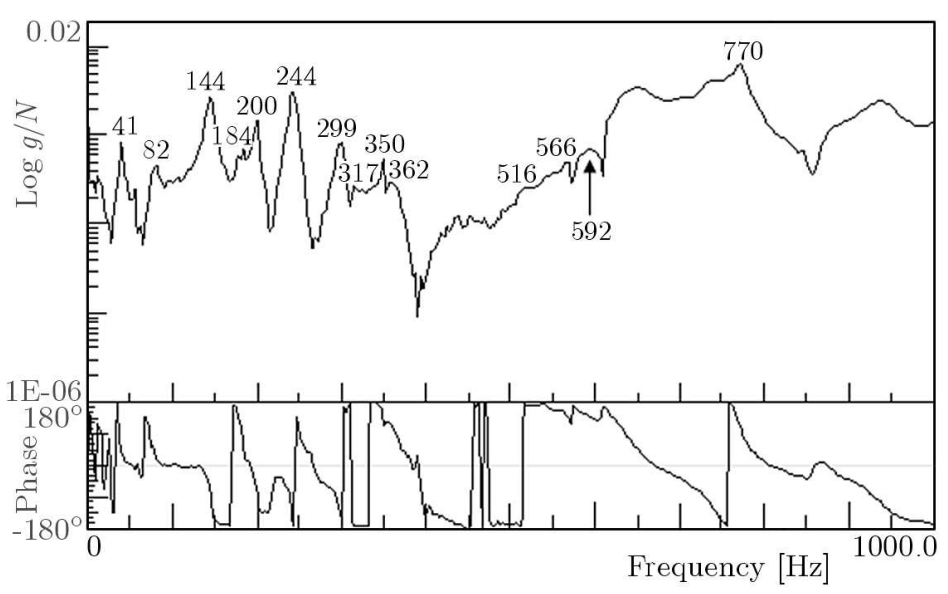

Fig. 4. Frequency response function of the fix ring for low frequencies

\subsubsection{Bearing modes}

The gear modes are identified by solving the eigen-value problem and using the impact test.

Figure 6 represents the frequency response function in the fixed ring with the hammer impact test.

Table 3 compares the natural frequencies determined from the impact test experiments and the numerical model in high frequencies.

Experiments show five natural frequencies whereas the numerical model shows three natural frequencies associated with three translational modes. The natural frequencies $1019 \mathrm{~Hz}$ and $1457 \mathrm{~Hz}$ which can be induced by the axial vibration appear only in the impulse test. The remaining natural frequencies agree within $7 \%$ as the maximum difference rate. 
Table 2. Natural frequencies from the experiments and numerical model in low frequencies

\begin{tabular}{|c|c|c|c|c|c|}
\hline Mode & Type & $\begin{array}{c}\text { Impulse } \\
\text { exp. [Hz] }\end{array}$ & $\begin{array}{c}\text { Numerical } \\
\text { model [Hz] }\end{array}$ & Multiplicity Error \\
{$[\%]$}
\end{tabular}

$192 \mathrm{~Hz}(\mathrm{~T})$

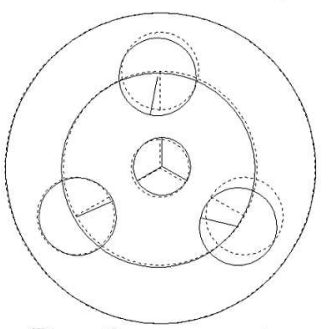

Reaction gear set

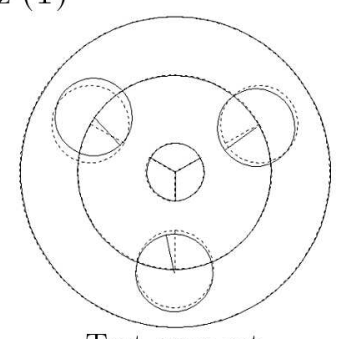

Test gear set

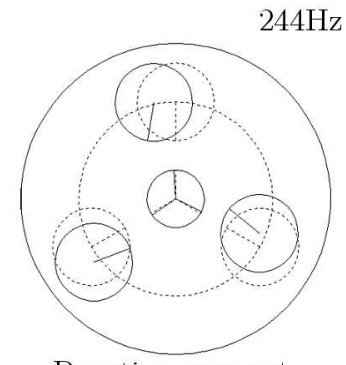

Reaction gear set

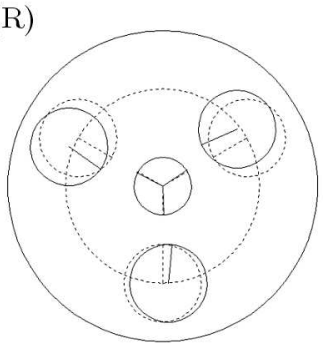

Test gear set

Fig. 5. Vibration modes in low frequencies

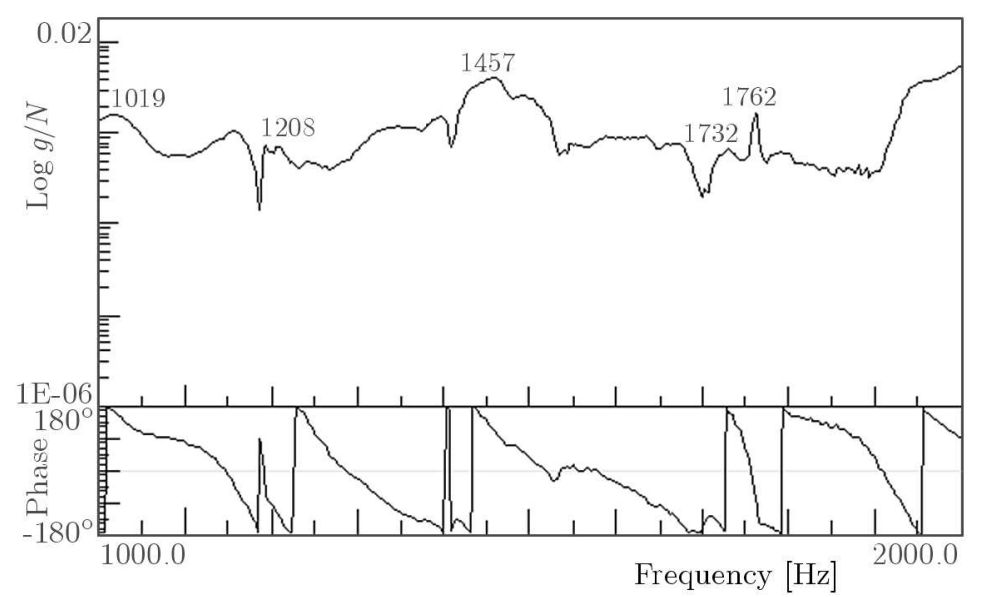

Fig. 6. Frequency response function of the fixed ring in high frequencies

Figure 7 shows that the carriers, the suns and the rings on the test and the reaction gear set have pure translation in the translation modes $1332 \mathrm{~Hz}$. 
Table 3. Natural frequencies from the experiments and numerical model in high frequencies

\begin{tabular}{|c|c|c|c|c|c|}
\hline Mode & Type & $\begin{array}{l}\text { Impulse } \\
\text { exp. }[\mathrm{Hz}]\end{array}$ & $\begin{array}{l}\text { Numerical } \\
\text { model }[\mathrm{Hz}]\end{array}$ & Multiplici & $\begin{array}{c}\text { Error } \\
\text { y }[\%] \\
\end{array}$ \\
\hline$\overline{16}$ & $=$ & $\bar{~} 1019$ & $=$ & - & $=$ \\
\hline 17 & $\mathrm{~T}$ & 1208 & 1232 & 2 & 2 \\
\hline 18 & - & 1457 & - & - & - \\
\hline 19 & $\mathrm{~T}$ & 1732 & 1847 & 2 & 7 \\
\hline 20 & $\overline{\mathrm{T}}$ & 1762 & 1888 & 2 & 7 \\
\hline
\end{tabular}

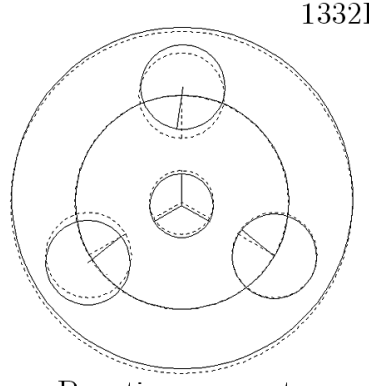

Reaction gear set

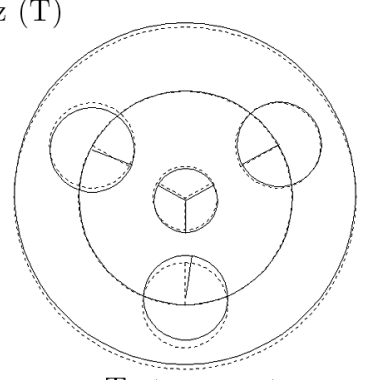

Test gear set

Fig. 7. Vibration modes in high frequencies

\subsection{Analysis of the distribution of modal kinetic energies and modal strain energies}

Computation of the modal strain energy and the modal kinetic energy distributions gives information on bodies sought to critical speeds (which excite the natural frequencies) in terms of dominant motion and deformation.

The total modal strain energy can be written as the sum of the strain energies of rotation and translation from each component

$$
E p_{\phi}=\frac{1}{2} \phi_{i}^{t} \widetilde{K} \phi_{i}=\sum E p_{\phi i j}+\sum E p_{\phi i j w}+\sum\left(E p_{\phi s u n}+E p_{\phi r i n}\right)
$$

where $E p_{\phi i j}$ and $E p_{\phi i j w}$ are the strain energy of bearing stiffness in the rotational and translational movement of the suns, carriers, rings and planets $(j=s, c, r, t, 1,2,3)$ in the test gear set and the reaction gear set $(i=t, r) . E p_{\phi s u n}$ and $E p_{\phi r i n}$ are the strain energy of sun-planets and planets-ring meshing in the test gear set and the reaction gear set.

The modal kinetic energy can also be written as the sum of the kinetic energies of rotation and translation from each component of the system

$$
E c_{\phi}=\frac{1}{2} w_{i}^{2} \phi_{i}^{t} M \phi_{i}=\sum E c_{\phi i j}+\sum E c_{\phi i j w}
$$

where $E c_{\phi i j}$ and $E c_{\phi i j w}$ are the kinetic energy in the rotational and translational movement of the suns, carriers, rings and planets $(j=s, c, r, t, 1,2,3)$ in the test gear set and the reaction gear set $(i=t, r)$.

\subsubsection{Teeth modes}

Figure 8 shows the distribution of modal kinetic energies in low frequencies.

In the $X$-axis the contribution of each degree of freedom in the total modal strain energy is represented. Details are given in Table 4.

The dominant movement of the translational mode $547 \mathrm{~Hz}$ is translation of the reaction ring along the $X$ direction. 

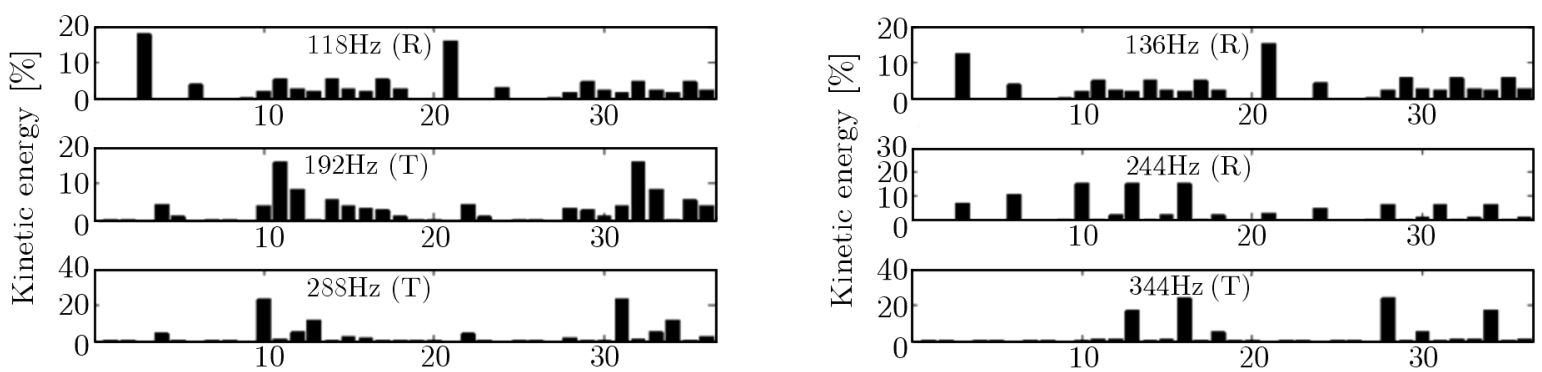

Fig. 8. Modal kinetic energies in low-frequencies

Table 4. The $X$-axis location of kinetic energies

\begin{tabular}{|l|l|l|l|}
\hline $1-2$ & Translations of reaction carrier & $19-20$ & Translations of test carrier \\
\hline 3 & Rotation of reaction carrier & 21 & Rotation of test carrier \\
\hline $4-5$ & Translations of reaction ring & $22-23$ & Translations of test ring \\
\hline 6 & Rotation of reaction ring & 24 & Rotation of test ring \\
\hline $7-8$ & Translations of reaction sun & $25-26$ & Translations of test sun \\
\hline 9 & Rotation of reaction sun & 27 & Rotation of test sun \\
\hline $10-11$ & Translations of reaction planet 1 & $28-29$ & Translations of test planet 1 \\
\hline 12 & Rotation of reaction planet 1 & 30 & Rotation of test planet 1 \\
\hline $13-14$ & Translations of reaction planet 2 & $31-32$ & Translations of test planet 2 \\
\hline 15 & Rotation of reaction planet 2 & 33 & Rotation of test planet 2 \\
\hline $16-17$ & Translations of reaction planet 3 & $34-35$ & Translations of test planet 3 \\
\hline 18 & Rotation of reaction planet 3 & 36 & Rotation of test planet 3 \\
\hline
\end{tabular}

Figure 9 shows the distribution of modal strain energies in low frequencies, where the $X$-axis is defined in Table 5 .
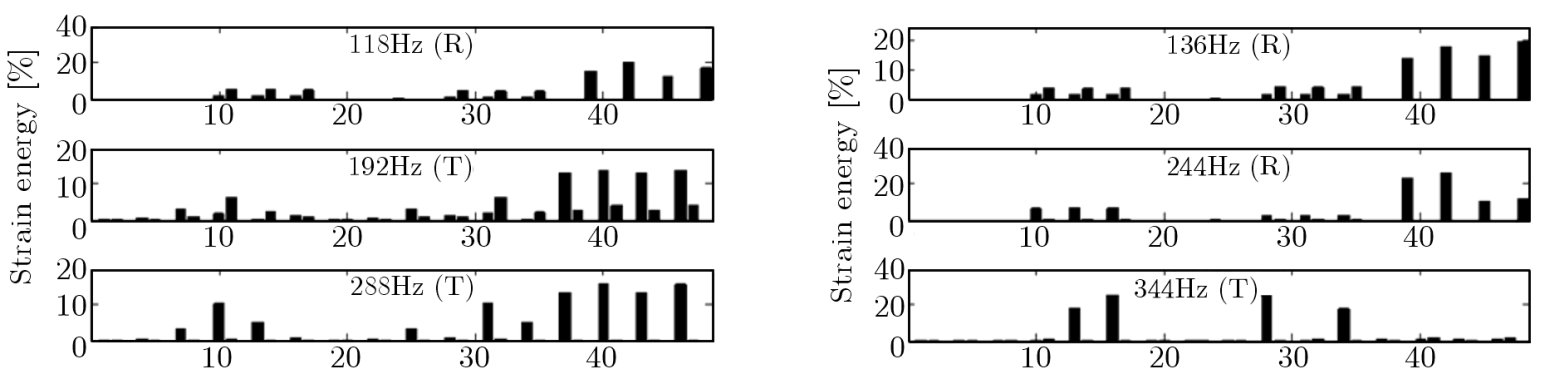

Fig. 9. Strain energy in low-frequencies

The dominant strain energy of the translational mode $547 \mathrm{~Hz}$ is located in the meshing between reaction ring-planet No. 3 .

Table 6 presents a resume of the modal dominant movement and the dominant strain energy in each low-frequency mode.

In the teeth modes which characterize the deflection in fixture components, the modes have significant strain energy in the teeth meshing sun-planets and ring-planets of the two stages. In fact, the stiffness of the meshing is higher than the stiffness of the shafts and bearing of the gears. So, the strain energies are bigger for the components which have higher stiffness. Also, those modes are predominantly characterized by motion of the individual planetary gear components, particularly the planet gears. 
Table 5. The $X$-axis location of strain energies

\begin{tabular}{|l|l|l|l|}
\hline $1-3$ & Bearing of reaction carrier & $25-27$ & Bearing of test sun \\
\hline $4-6$ & Bearing of reaction ring & $28-30$ & Bearing of test planet 1 \\
\hline $7-9$ & Bearing of reaction sun & $31-33$ & Bearing of test planet 2 \\
\hline $10-12$ & Bearing of reaction planet 1 & $34-36$ & Bearing of test planet 3 \\
\hline $13-15$ & Bearing of reaction planet 2 & $37-39$ & Meshing reaction ring-planets \\
\hline $16-18$ & Bearing of reaction planet 3 & $40-42$ & Meshing reaction sun-planets \\
\hline $19-21$ & Bearing of test carrier & $43-45$ & Meshing test ring-planets \\
\hline $22-24$ & Bearing of test ring & $46-48$ & Meshing test sun-planets \\
\hline
\end{tabular}

Table 6. Dominant motion and dominant strain energy in low-frequencies

\begin{tabular}{|c|c|c|}
\hline Frequencies & Modal dominant movement & Dominant strain energy \\
\hline $118 \mathrm{~Hz}(\mathrm{R})$ & Rotation of reaction carrier & Meshing reaction sun-planet 3 \\
\hline $136 \mathrm{~Hz}(\mathrm{R})$ & Rotation of test carrier & Meshing test sun-planet 3 \\
\hline \multirow[t]{2}{*}{$192 \mathrm{~Hz}(\mathrm{~T})$} & Translation of reaction planet $1(X)$ & Meshing reaction sun-planet 1 \\
\hline & Translation of test planet $2(Y)$ & Meshing test sun-planet 1 \\
\hline $244 \mathrm{~Hz}(\mathrm{R})$ & Rotation of all test planet $(X)$ & Meshing reaction sun-planet 3 \\
\hline \multirow[t]{2}{*}{$288 \mathrm{~Hz}(\mathrm{~T})$} & Translation of reaction planet $1(X)$ & Meshing reaction sun-planet 1 \\
\hline & Translation of test planet $2(X)$ & Meshing test sun-planet 1 \\
\hline \multirow[t]{2}{*}{$344 \mathrm{~Hz}(\mathrm{~T})$} & Translation of reaction planet $3(X)$ & Bearing of reaction planet $3(X)$ \\
\hline & Translation of test planet $1(X)$ & Bearing of test planet $3(X)$ \\
\hline $376 \mathrm{~Hz}(\mathrm{R})$ & Rotation of reaction carrier & Meshing reaction ring-planet 3 \\
\hline $387 \mathrm{~Hz}(\mathrm{R})$ & Rotation of test carrier & Meshing test ring-planet 3 \\
\hline \multirow[t]{2}{*}{$536 \mathrm{~Hz}(\mathrm{R})$} & Translation of reaction ring $(X)$ & Meshing reaction ring-planet 1 \\
\hline & Translation of test ring $(X)$ & Meshing test sun-planet 2 \\
\hline $547 \mathrm{~Hz}(\mathrm{R})$ & Rotation of reaction ring & Meshing reaction ring-planet 3 \\
\hline $550 \mathrm{~Hz}(\mathrm{R})$ & Rotation of test ring & Meshing test ring-planet 3 \\
\hline
\end{tabular}

$(X)-X$ direction, $(Y)-Y$ direction

\subsubsection{Bearing modes}

Figure 10 shows the distribution of modal kinetic energies in high frequencies. The $X$-axis is defined in Table 4 .

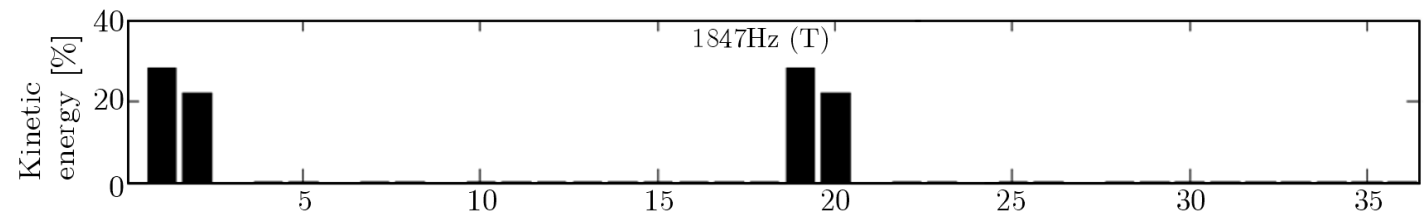

Fig. 10. Kinetic energy in high-frequencies

The dominant motion in the translational mode $1867 \mathrm{~Hz}$ is the translation of the reaction sun in the $Y$ direction.

Figure 11 shows the distribution of modal strain energies in low frequencies. The $X$-axis is defined in Table 5 .

The dominant strain energy in the translational mode $2527 \mathrm{~Hz}$ is the bearing of the test carrier in the $Y$ direction.

Table 7 presents a resume of the modal dominant movement and the dominant strain energy in each high-frequency mode. 


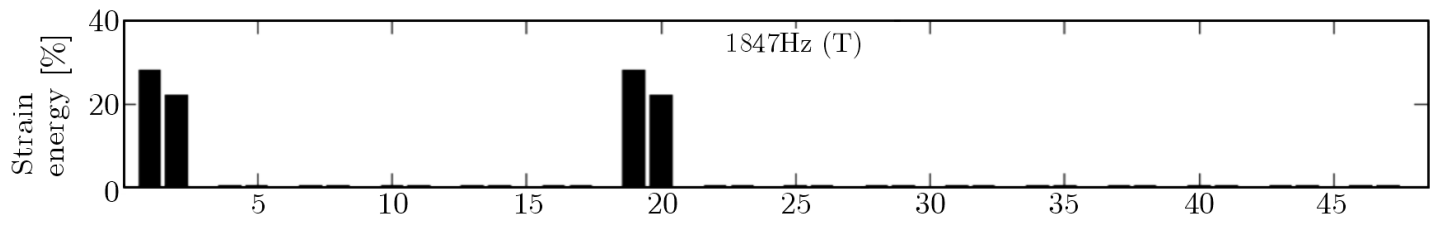

Fig. 11. Strain energy in high-frequencies

Table 7. Dominant motion and dominant strain energy in high-frequencies

\begin{tabular}{|c|l|l|}
\hline Frequencies & \multicolumn{1}{|c|}{ Modal dominant movement } & \multicolumn{1}{|c|}{ Dominant strain energy } \\
\hline \hline \multirow{2}{*}{$1232 \mathrm{~Hz}(\mathrm{~T})$} & Translations of reaction ring $(Y)$ & Meshing reaction ring-planet 2 \\
\cline { 2 - 3 } & Translations of test planet $1(X)$ & Meshing test ring-planet 2 \\
\hline \multirow{2}{*}{$1847 \mathrm{~Hz}(\mathrm{~T})$} & Translations of reaction carrier $(X)$ & Bearing of reaction carrier $(X)$ \\
\cline { 2 - 3 } & Translations of test carrier $(X)$ & Bearing of test carrier $(X)$ \\
\hline \multirow{2}{*}{$1888 \mathrm{~Hz}(\mathrm{~T})$} & Translations of reaction carrier $(X)$ & Bearing of reaction carrier $(X)$ \\
\cline { 2 - 3 } & Translations of test carrier $(X)$ & Bearing of test carrier $(X)$ \\
\hline
\end{tabular}

Table 7 shows bearing modes which are characterized by the strain and kinetic energies of the carriers $(1847 \mathrm{~Hz}$ and $1888 \mathrm{~Hz})$.

In general, the significant strain energies in low frequencies are in gears teeth where the mesh stiffness is higher than the stiffness of the shafts and bearing, and the motion of gears has the highest kinetic energy. However, for the same condition, the dominant movement in high frequencies is the movement of carriers which have also the dominant strain energies.

\section{Conclusions}

A modal analysis has been performed on a back-to-back planetary gear using two approaches: model based and experimental study. Concerning the modelling approach, a mathematical model of the gear system has been proposed. Solving the eigenvalue problem allowed recovering the modal characteristics of the transmission. It has been found that the natural frequencies can be divided into two main modes: rotational modes and translational modes. The experimental modal investigation done on a test rig confirmed this classification. When looking to the modal kinetic and strain energy distributions, another classification emerges according to the dominant energy in the system for each natural frequency. In fact, teeth modes and bearing modes have been highlighted. The selection of parameters in the model has been optimized to get results as close as possible to the experimental results done by the hammer test. The adopted model in the paper considers only 3 degrees of freedom per component. Obviously, it will not be possible to get low percentage error for all the computed natural frequencies. Further modal analysis investigations will be done in future works with a model considering 6 degrees of freedom per component. Such study can help designers to avoid critical frequencies on the system by changing parameters of the system. It allows one also to localise critical components susceptible to damage when crossing natural frequencies.

\section{Acknowledgements}

This paper was financially supported by the Tunisian-Spanish Joint Project No. A1/037038/11.

The authors would like also to acknowledge project "Development of methodologies for the simulation and improvement of the dynamic behavior of planetary transmissions DPI2013-44860" funded by the Spanish Ministry of Science and Technology.

Acknowledgment to the University of Cantabria cooperation project for doctoral training of University of Sfax's students. 


\section{A. Appendix}

The matrix mass is defined

$$
\mathbf{M}=\operatorname{diag}\left(\mathbf{M}_{c r}, \mathbf{M}_{r r}, \mathbf{M}_{s r}, \mathbf{M}_{1 r}, \mathbf{M}_{2 r}, \mathbf{M}_{3 r}, \mathbf{M}_{c t}, \mathbf{M}_{r t}, \mathbf{M}_{s t}, \mathbf{M}_{1 t}, \mathbf{M}_{2 t}, \mathbf{M}_{3 t}\right)
$$

where

$$
\begin{aligned}
& \mathbf{M}_{i j}=\operatorname{diag}\left(m_{i j}, m_{i j}, I_{i j} / r_{i j}^{2}\right) \quad i=c, r, s, 1,2,3 \quad j=r, t \\
& \mathbf{G}=\operatorname{diag}\left(\mathbf{G}_{c r}, \mathbf{G}_{r r}, \mathbf{G}_{s r}, \mathbf{G}_{1 r}, \mathbf{G}_{2 r}, \mathbf{G}_{3 r}, \mathbf{G}_{c t}, \mathbf{G}_{r t}, \mathbf{G}_{s t}, \mathbf{G}_{1 t}, \mathbf{G}_{2 t}, \mathbf{G}_{3 t}\right) \\
& \mathbf{G}_{i j}=\left[\begin{array}{ccc}
0 & -2 m_{i j} & 0 \\
2 m_{i j} & 0 & 0 \\
0 & 0 & 0
\end{array}\right] \quad i=c, r, s, 1,2,3 \quad j=r, t \\
& \mathbf{K}_{\Omega}=\operatorname{diag}\left(\mathbf{K}_{\Omega_{r}}, \mathbf{K}_{\Omega_{t}}\right) \\
& \mathbf{K}_{\Omega_{r}}=\operatorname{diag}\left(m_{c r}, m_{c r}, 0, m_{r r}, m_{r r}, 0, m_{s r}, m_{s r}, 0, m_{p r}, m_{p r}, 0, m_{p r}, m_{p r}, 0, m_{p r}, m_{p r}, 0\right) \\
& \mathbf{K}_{\Omega_{t}}=\operatorname{diag}\left(m_{c t}, m_{c t}, 0, m_{r t}, m_{r t}, 0, m_{s t}, m_{s t}, 0, m_{p t}, m_{p t}, 0, m_{p t}, m_{p t}, 0, m_{p t}, m_{p t}, 0\right)
\end{aligned}
$$

The bearing stiffness matrix

$$
\begin{aligned}
& \mathbf{K}_{b}=\operatorname{diag}\left(\mathbf{K}_{c r b}, \mathbf{K}_{r r b}, \mathbf{K}_{s r b}, \mathbf{0}, \mathbf{0}, \mathbf{0}, \mathbf{K}_{c t b}, \mathbf{K}_{r t b}, \mathbf{K}_{s t b}, \mathbf{0}, \mathbf{0}, \mathbf{0}\right) \\
& \mathbf{K}_{i j b}=\operatorname{diag}\left(\mathbf{K}_{i j x}, \mathbf{K}_{i j y}, \mathbf{K}_{i j u}\right) \quad i=c, r, s, 1,2,3 \quad j=r, t \\
& \mathbf{K}_{m}=\left[\begin{array}{cc}
\mathbf{K}_{m r} & \mathbf{0} \\
\mathbf{0} & \mathbf{K}_{m t}
\end{array}\right]+\mathbf{K}_{c} \\
& \mathbf{K}_{m}=\left[\begin{array}{cccccc}
\sum \mathbf{K}_{c 1}^{n} & \mathbf{0} & \mathbf{0} & \mathbf{K}_{c 2}^{1} & \mathbf{K}_{c 2}^{2} & \mathbf{K}_{c 2}^{3} \\
\mathbf{0} & \sum \mathbf{K}_{r 1}^{n} & \mathbf{0} & \mathbf{K}_{r 2}^{1} & \mathbf{K}_{r 2}^{2} & \mathbf{K}_{r 2}^{3} \\
\mathbf{0} & \mathbf{0} & \sum \mathbf{K}_{s 1}^{n} & \mathbf{K}_{s 1}^{1} & \mathbf{K}_{s 1}^{2} & \mathbf{K}_{s 1}^{3} \\
\mathbf{K}_{c 2}^{1} & \mathbf{K}_{r 2}^{1} & \mathbf{K}_{s 1}^{1} & \mathbf{K}_{p p}^{1} & \mathbf{0} & \mathbf{0} \\
\mathbf{K}_{c 2}^{2} & \mathbf{K}_{r 2}^{2} & \mathbf{K}_{s 1}^{2} & \mathbf{0} & \mathbf{K}_{p p}^{2} & \mathbf{0} \\
\mathbf{K}_{c 2}^{3} & \mathbf{K}_{r 2}^{3} & \mathbf{K}_{s 1}^{3} & \mathbf{0} & \mathbf{0} & \mathbf{K}_{p p}^{3}
\end{array}\right] \\
& \mathbf{K}_{p p}^{n}=\mathbf{K}_{c 3}^{n}+\mathbf{K}_{r 3}^{n}+\mathbf{K}_{s 3}^{n} \\
& \mathbf{K}_{c 1}^{n}=k_{p n}\left[\begin{array}{ccc}
1 & 0 & -\sin \Psi_{n} \\
0 & 1 & \cos \Psi_{n} \\
-\sin \Psi_{n} & \cos \Psi_{n} & 1
\end{array}\right] \\
& \mathbf{K}_{c 2}^{n}=k_{p n}\left[\begin{array}{ccc}
-\cos \Psi_{n} & \sin \Psi_{n} & 0 \\
-\sin \Psi_{n} & -\cos \Psi_{n} & 0 \\
0 & -1 & 0
\end{array}\right] \\
& \mathbf{K}_{c 3}^{n}=\operatorname{diag}\left(k_{p n}, k_{p n}, 0\right) \\
& \mathbf{K}_{r 1}^{n}=k_{r n}\left[\begin{array}{ccc}
\sin ^{2} \Psi_{r n} & -\cos \Psi_{r n} \sin \Psi_{r n} & -\sin \Psi_{r n} \\
-\cos \Psi_{r n} \sin \Psi_{r n} & \cos ^{2} \Psi_{r n} & \cos \Psi_{r n} \\
-\sin \Psi_{r n} & \cos \Psi_{r n} & 1
\end{array}\right] \\
& \mathbf{K}_{r 2}^{n}=k_{r n}\left[\begin{array}{ccc}
-\sin \Psi_{r n} \sin \alpha_{r} & \sin \Psi_{r n} \cos \alpha_{r} & \sin \Psi_{r n} \\
\cos \Psi_{r n} \sin \alpha_{r} & -\cos \Psi_{r n} \cos \alpha_{r} & -\cos \Psi_{r n} \\
\sin \alpha_{r} & -\cos \alpha_{r} & -1
\end{array}\right]
\end{aligned}
$$




$$
\begin{aligned}
& \mathbf{K}_{r 3}^{n}=k_{r n}\left[\begin{array}{ccc}
\sin ^{2} \alpha_{r} & -\cos \alpha_{r} \sin \alpha_{r} & -\sin \alpha_{r} \\
-\cos \alpha_{r} \sin \alpha_{r} & \cos ^{2} \alpha_{r} & \cos \alpha_{r} \\
-\sin \alpha_{r} & \cos \alpha_{r} & 1
\end{array}\right] \\
& \mathbf{K}_{s 1}^{n}=k_{s n}\left[\begin{array}{ccc}
\sin ^{2} \Psi_{s n} & -\cos \Psi_{s n} \sin \Psi_{s n} & -\sin \Psi_{s n} \\
-\cos \Psi_{s n} \sin \Psi_{s n} & \cos ^{2} \Psi_{s n} & \cos \Psi_{s n} \\
-\sin \Psi_{s n} & \cos \Psi_{s n} & 1
\end{array}\right] \\
& \mathbf{K}_{s 2}^{n}=k_{s n}\left[\begin{array}{ccc}
\sin \Psi_{s n} \sin \alpha_{s} & \sin \Psi_{s n} \cos \alpha_{s} & -\sin \Psi_{s n} \\
-\cos \Psi_{s n} \sin \alpha_{s} & -\cos \Psi_{s n} \cos \alpha_{s} & \cos \Psi_{s n} \\
-\sin \alpha_{s} & -\cos \alpha_{s} & 1
\end{array}\right] \\
& \mathbf{K}_{s 3}^{n}=k_{s n}\left[\begin{array}{ccc}
\sin \alpha_{s} \alpha_{s} & \cos \alpha_{s} \sin \alpha_{s} & -\sin \alpha_{s} \\
\cos \alpha_{s} \sin \alpha_{s} & \cos \alpha_{s} \alpha_{s} & \cos \alpha_{s} \\
-\sin \alpha_{s} & -\cos \alpha_{s} & 1
\end{array}\right] \\
& \Psi_{s n}=\Psi_{n}-\alpha_{s} \Psi_{r n}=\Psi_{n}+\alpha_{r}
\end{aligned}
$$

\section{References}

1. August R., Kasuba R., 1986, Torsional vibrations and dynamic loads in a basic planetary gear system, Journal of Vibration, Acoustics, Stress and Reliability in Design, 108, 348-353

2. Bartelmus W., 2001, Gearbox dynamic modelling, Journal of Theoretical and Applied Mechanics, 39, 4, 989-999

3. Botman M., 1976, Epicyclic gear vibrations, Journal of Engineering for Industry, 98, 811-815

4. Bu Z., LIU G., Wu L., 2012, Modal analyses of herringbone planetary gear train with journal bearings, Mechanism and Machine Theory, 54, 99-115

5. Cooley C.G., Parker R.G., 2012, Vibration properties of high-speed planetary gears with gyroscopic effects, Journal of Vibration and Acoustics, 134, 061014

6. Cooley C.G., Parker R.G., 2013, Mechanical stability of high-speed planetary gears, International Journal of Mechanical Sciences, 69, 59-71

7. Cunliffe F., Smith J.D., Welbourn D.B., 1974, Dynamic tooth loads in epicyclic gears, Journal of Engineering for Industry, 95, 578-584

8. Ericson T.M., PArker R.G., 2013, Planetary gear modal vibration experiments and correlation against lumped-parameter and finite element models, Journal of Sound and Vibration, 332, 2350-2375

9. Eritenel T., Parker R.G., 2009, Modal properties of three-dimensional helical planetary gears, Journal of Sound and Vibration, 325, 397-420

10. Guo Y., PArker R.G., 2010, Purely rotational model and vibration modes of compound planetary gears, Mechanism and Machine Theory, 45, 365-377

11. Kahraman A., 1993, Planetary gear train dynamics, Journal of Mechanical Design, 116, 713-720

12. Kahraman A., 1994, Natural modes of planetary gear trains, Journal of Sound and Vibration, 173, 125-130

13. Kahraman A., ViJayAKAR S.M., 2001, Effect of internal gear flexibility on the quasi-static behavior of a planetary gear set, Journal of Mechanical Design, 123, 408-415

14. Kiracofe D.R., Parker R.G., 2007, Structured vibration modes of general compound planetary gear systems, Journal of Vibration and Acoustics, 129, 1-16

15. Lin J., PARKer R.G., 1999, Analytical characterization of the unique properties of planetary gear free vibration, Journal of Vibration and Acoustics, 121, 316-321 
16. Lin J., PARKer R.G., 2000, Structured vibration characteristics of planetary gears with unequally spaced planets, Journal of Sound and Vibration, 233, 921-928

17. Łuczko J., 2008, Chaotic vibrations in gear mesh systems, Journal of Theoretical and Applied Mechanics, 46, 4, 879-896

18. PARker R.G, Wu X., 2010, Vibration modes of planetary gears with unequally spaced planets and an elastic ring gear, Journal of Sound and Vibration, 329, 2265-2275

19. Singh A., Kahraman A., Ligata H., 2008, Internal gear strains and load sharing in planetary transmissions: model and experiments, Journal of Mechanical Design, 130, 072602

20. Sondkar P., Kahraman A., 2013, A dynamic model of a double-helical planetary gear set, Mechanism and Machine Theory, 70, 157-174

21. TAnna R.P., Lim T.C., 2004, Modal frequency deviations in stimating ring gear modes using smooth ring solutions, Journal of Sound and Vibration, 269, 1099-1110

22. Toda A., Botman M., 1979, Planet indexing in planetary gears for minimum vibration, $A S M E$ Design Engineering Technical Conference, A80-15730, ASME, St. Louis

23. Velex P., Randrianarivo L., Pichon V., Wittman R., 1994, Dynamic behavior of epicyclic trains: experimental and numerical analyses, Proceedings of the International Gearing Conference, Newcastle, 265-269

24. Wojnarowski J., Kopeć J., Zawiślak S., Gears and graphs, Journal of Theoretical and Applied Mechanics, 44, 1, 139-162

25. Wu X., PARker R.G., 2008, Modal properties of planetary gears with an elastic continuum ring gear, Journal of Applied Mechanics, 75, 1-10 